\title{
Pediatric clinical trials: a US perspective
}

This article was published in the following Dove Press journal:

Open Access Journal of Clinical Trials

26 November 2014

Number of times this article has been viewed

\section{Matthew J Oelstrom' Margo L Hoover-Regan ${ }^{2}$ \\ 'Children's Hospital of Wisconsin, Milwaukee, WI, USA; 'Division of Pediatric Hematology and Oncology, The University of Wisconsin School of Medicine and Public Health, Madison, WI, USA}

Background: Since the scientific revolution, systematic child-subject experimentation has evolved, with regard not only to scientific methodology but also to appreciation of the vulnerability of pediatric subjects. Permission-assent or informed consent policies serve to protect pediatric subjects. Unfortunately, child-subject and parent-surrogate understanding of research is not satisfactory.

Methods: The PubMed, Essential Evidence Plus, and CINAHL Plus databases were searched for literature on informed consent, permission, and assent in pediatric clinical trials. Articles with an emphasis on the parents' and subjects' understanding of clinical trials were selected for review and summary.

Results: Seventy unique articles satisfied the search criteria. Each article was reviewed thoroughly for information about the informed consent process, parent (and, in some cases, child-subject) understanding of the nature of specific pediatric clinical trials, and procedures used or proposed to improve understanding of clinical research by subjects and/or their parents.

Conclusion: Many parent-surrogates and child-subjects do not adequately understand clinical research. Parents and children often fail to understand randomization, especially as it relates to the principle of clinical equipoise. Children have additional difficulty with the nature of placebos and with right to withdraw from research at any time. Future research should prospectively evaluate interventions such as "staged consent," public education, medical trainee education, and alternative information-delivery methods, which are not yet known to consistently affect understanding.

Keywords: informed consent, permission-assent, subject understanding

\section{Background}

Scientific research on child-subjects was born concomitantly with other systematic research during the Scientific Revolution. In his cowpox research, Jenner employed his own and his gardener's sons as subjects, and other 18th-century child-subjects were inoculated with pathogens and deprived of nutrients to study the natural history of diseases such as syphilis and scurvy. ${ }^{1}$ Pasteur piloted the rabies vaccine on 9-yearold Joseph Meister after Meister was bitten by a rabid dog in $1885 .^{2}$ One Austrian researcher of the late 19 th century used human foundlings from the local orphanage as subjects because they were "less expensive than animals."

As the 19th century public became aware of occasional negative exploitation in pediatric research, they recognized the need to protect this vulnerable population from unscrupulous researchers. In 1900, the Prussian Minister of Religious, Educational, 
and Medical Affairs explicitly forbade research on children and other vulnerable populations. Specifically, the directive stated that medical interventions other than those deemed for diagnosis, therapy or immunization were to be prohibited if the patient was a minor; if the patient had not consented to the intervention; or if potential risks of the intervention had not been explained. ${ }^{4}$

While child research never ceased during the 20th century, a large sector of the scientific community frowned on it. ${ }^{1}$

Investigators who did undertake child research in the early 20th century were expected to protect their subjects from inordinate risks of research and to require proxy informed consent from parents, parallel to expectations for research on adults. Contrary to the 1900 Prussian regulations, 1931 German policies did allow for pediatric research. The [Third] Reich Health Council, an advisory body that included German doctors, pharmacists, and governmental appointees, recommended that research on children be acceptable provided that parents gave proxy-consent and that the proposed research did not endanger the child or minor. ${ }^{2}$

In Germany (despite the Council's recommendations) and elsewhere, investigator deviation from moral norms and laws resulted in further regulation of research. Notoriously, Karl Brandt and 15 other German physicians were convicted at Nuremberg of crimes against humanity for their oversight of systematic human experimentation in German concentration camps, including research on children. Even though it did not specifically address pediatric research, the 1947 Nuremberg Code became the basis of later regulations requiring informed consent in medical research. ${ }^{2}$ For instance, it was an important influence of the 1964 Declaration of Helsinki, which reaffirmed that parental proxy consent could substitute for informed consent in cases of pediatric research.

Despite these international declarations, several highly scrutinized child research projects in the United States provoked public outcry. Beecher became a whistleblower when his report on 22 exploitative studies was published in the New England Journal of Medicine. ${ }^{5}$

One of Beecher's questionable projects took place at the Willowbrook State School during the 1950s and 1960s. Willowbrook was an institution on Staten Island for developmentally delayed children. Parents of incoming residents were given the opportunity to include their children in a study that involved inoculating the children with strains of the hepatitis A virus. The investigators rationalized that greater than $90 \%$ would likely be infected anyway, given their institutionalization at Willowbrook. The Willowbrook research was fundamental in the characterization of the distinction between hepatitis $\mathrm{A}$ and $\mathrm{B}$ and in the development of the hepatitis B vaccine by the same researchers. ${ }^{6}$

Despite the actus reus of injecting institutionalized children with known pathogens, the Willowbrook researchers were praised for their progressive parental permission process, which involved an initial face-to-face meeting with a social worker, a presentation by the investigators, a 2-week waiting period for parents to discuss the study with their family physician, and a signed permission document, from which parents were informed they could withdraw at any time. ${ }^{1,2}$

During the same era, 74 members of the "science club" at the Fernald School for "feeble-minded" boys in Massachusetts were fed radioactive isotopes in their oatmeal by researchers at the Massachusetts Institute of Technology as part of the United States Atomic Energy Commission Human Radiation Experiments. In this case, parental permission was poor. The letter to parents describing the study stated that the subjects would receive a "special diet" and implied that the diet would be healthier than the standard resident diet. There was no opportunity for questions; parents were asked to sign and return a slip affirming they did not object to the study. ${ }^{2}$

Both the Willowbrook and Fernald experiments demonstrated potential problems with scientific research on vulnerable populations; they deepened the scientific community's aversion to child research. In 1977, the American Congress ordered the National Commission for Protection of Human Subjects of Biomedical and Behavioral Research to issue recommendations for the protection of childsubjects. These recommendations were the first to call for child assent as well as parental permission for pediatric clinical research. ${ }^{7}$

Despite regulatory support for pediatric research, the mid-20th century medical literature actually contains a paucity of pediatric studies because of the idea that the innocence and vulnerability of childhood demands that children be safeguarded from the risks associated with scientific research. The dearth of pediatric research led to a lack of advancement in the treatment of children, who "remained 'therapeutic orphans,' with the usage of most drugs in children based upon extrapolation of adult data to younger groups." Such extrapolation sometimes resulted in the application of dangerous and ineffective treatments to children, especially via inappropriate drug dosages based on adult models. ${ }^{1}$

A late-20th century appreciation for the unique physiology of children and the desire to provide them (like women 
and ethnic minorities) with equally unique and appropriate treatments dismantled the myth that children should be excluded from research. ${ }^{8}$ Pediatric clinical research that respects the dignity of the person, just as adult research aims to do, was advocated. In 1989, Bartholome encouraged pediatric research and described parental permission and child assent as the paradigm "informed consent" process for pediatric study, a position adopted by the American Academy of Pediatrics Committee on Bioethics in 1995.9,10 The change in the scientific community's disposition was further reflected in the National Institutes of Health's 1998 requirement that applicants justify exclusion of children from study designs rather than inclusion. ${ }^{11}$ Well into the 21 st century, experimentation on children continues to increase, especially in children with cancer, over $90 \%$ of whom are treated by members of the research-oriented Children's Oncology Group. ${ }^{2,12}$

The Federal Common Rule requires parental permission and child assent as the two components of informed consent for pediatric research. ${ }^{13}$ Further, potential risks to children must be identified and minimized. For nontherapeutic research, the risks must not be greater than a "minor increase over minimal risk" (ie, slightly more than the risks encountered in the normal, everyday lives of healthy children or the risks incurred in a routine visit to a physician). For therapeutic research, the risks must be "justified by anticipated benefit to the subjects" if they are greater than a "minor increase over minimal risk." Researchers and individual institutions are expected to weigh the risks associated with each proposed research study and determine if it is consistent with the federal requirement.

The terms "assent" and "permission" are used preferentially throughout this article where other authors might employ "consent." Used together in the context of pediatric research, "assent" and "permission" are understood to approximate "consent."

By law, the "when" and "how" of permission and assent are largely left to individual institutions, but physicianinvestigators are expected to optimize the permission and assent processes by presenting the risks and benefits of the proposed research and ensuring parental and child understanding of research protocols - to the extent possible - and by ensuring the meaningfulness (and not mere formality) of the processes. ${ }^{7,14,15}$

\section{Methods}

The PubMed, Essential Evidence Plus, and CINAHL Plus databases were used to identify relevant English-language literature. Search terms included consent, informed consent, permission, assent, proxy-consent, pediatric research, pediatric clinical trials, vulnerable population, understanding of research, child understanding, and parental understanding. Titles and abstracts with an emphasis on information about the informed consent process in pediatrics, parent (and, in some cases, child-subject) understanding of pediatric clinical trials, and interventions used or proposed to improve understanding of pediatric research by subjects or their parents were selected for review and summary. Further references were identified by reviewing the citations in each of the various identified papers. References from any date before the final literature search in July 2009 were considered.

\section{Results and analysis}

Seventy unique titles satisfied the search criteria. The degree of scientific validity of the papers varied significantly. Because of the relative paucity of information on these topics, opinion pieces or editorials were included along with retrospective and prospective reviews and randomizedcontrolled trials. Nine articles were excluded when fulltext review demonstrated that they were not relevant to permission, assent, or understanding in pediatric clinical trials. Thus, 61 of the 70 titles were accepted, and recurrent themes were identified and are described below. Given that additional search terms and databases (eg, Google Scholar) did not yield additional unique works for consideration, the selected references are believed to represent a broad base of the literature on the understanding of assent and permission in pediatric research.

\section{Discussion}

\section{Practical and demographic influences on parental understanding}

There was a wide range of parental understanding of pediatric clinical trials in the reviewed articles. Tait et al observed variable understanding at a Midwestern institution in 2003. ${ }^{16,17}$ That report included 102 children and 505 parents, and retrospectively assessed understanding of ongoing anesthesia or surgery studies that had been offered at the time of the subjects' presentation for elective procedures. Tait et al did appreciate a positive association between understanding and parental age, child age, and parental education level. Similarly, Simon et al ${ }^{18}$ reported on a group of 140 permission-assent conferences that were initially described by Kodish et al. ${ }^{19}$ Simon et al ${ }^{18}$ found that higher parental education levels and Caucasian ethnicity were associated with better understanding of the concept of randomization. 
In a similar analysis of the same 140 pediatric leukemia patients offered randomized clinical trials as described by Kodish et al, Hazen et al report that parents with higher socioeconomic status were more likely to both read the permission documents and better understand the elements of permission. ${ }^{20}$ These authors report that parental age and sex were not correlated with understanding. In 2004, Kodish et al interviewed parents after 137 permission-assent conferences for leukemia treatment trials at six American academic medical centers. They established that $50 \%$ of parents did not understand randomization, and parents of minority race or lower socioeconomic status were significantly less likely to understand randomization $\left(P<0.001\right.$ for each) ${ }^{19}$

Based on the experience of these authors, high understanding often correlated with older age, higher educational achievement, and higher socioeconomic status.

\section{Therapeutic misconception}

The previously discussed variables are easily quantifiable and represent only some of the many uncontrolled factors that affect how subjects understand clinical trials. The earliest articles on the understanding of research considered adults participating in clinical trials and took place before many of the current requirements for informed consent were established. The most common type of misunderstanding was termed the "therapeutic misconception" by Appelbaum et al in $1982 .{ }^{21}$ Therapeutic misconception is the false belief that the main purpose of a clinical trial is to treat the subject's illness; it is often revealed when subjects express the conviction that the researcher chooses the best possible treatment for the subject based on his or her unique characteristics, rather than by randomization.

Edwards et al reviewed the literature on the understanding of clinical equipoise and found it regularly deficient. ${ }^{22}$ They warn that patients cannot truly understand the nature of research if they do not appreciate one of its most fundamental procedures, randomization. In an essay for pediatric hematologist-oncologists about how to facilitate permission and assent, Massimo et al described randomization as "an unsurmountable enemy" to parents of children in randomized trials. ${ }^{23}$ In an update on current research into improving permission and assent, Barfield stated, "central concepts in pediatric research such as randomization [...] are not uniformly understood by parents or older pediatric patients. ${ }^{24}$ Equipoise is an essential feature of the randomized clinical trial and a feature that Applebaum et al feel the subjects should understand to be truly informed about their clinical trials. ${ }^{21}$
Some studies specifically identified how well parents understood studies in which they had permitted their children to participate. Snowdon et al interviewed the parents of 21 children 12 to 25 months after they had participated in a neonatal study of extracorporeal membrane oxygenation. ${ }^{25}$ Surprisingly, parents of only eleven of the 21 children appreciated that their children had been randomly assigned to a treatment group, despite randomization presumptively being described in the permission form. Unfortunately, Snowdon et al did not procure the initial permission for the clinical trial, and they did not have access to all versions of the permission documents. Of the eleven parents who truly appreciated random assignment, only the parents of four children identified a methodological purpose of randomization (ie, to eliminate variables between treatment groups). The parents of three other children stated that the purpose of randomization was to spare the doctor from having to make the difficult decision of how to treat the baby. Another set of parents thought that randomization was necessary because of resource limitations in the British National Health Service. For them, randomization was a means of rationing health care; the government was offering the experimental treatment on a "trial basis."

Even though this study was limited by a small sample size and uncertainty about what had actually been explained to parents at the time of the trial, Snowdon et al made three other interesting observations about the informants. ${ }^{25}$ First, many of the ten parents who agreed that treatment allocation had been "randomized" actually described nonrandom allocation procedures in which the investigators chose which babies to assign to the treatment and control arms based on individual characteristics of the baby, such as blood oxygen content. Second, parents frequently used the name of the study and the name of the experimental treatment interchangeably, suggesting that they did not appreciate the control arm of the study as an important aspect of the experimental process. Additionally, almost all the parents denied clinical equipoise between the treatment arms. They insisted that the experimental treatment was superior to the control intervention. Factors influencing the parents' attitudes are unknown in this small, qualitative study.

Vitiello et al hypothesized that the therapeutic misconception would be more likely among the parents of subjects who had been in long-term fiduciary relationships with researchers before beginning a clinical trial. ${ }^{26}$ In a pediatric, double-blinded, placebo-controlled trial of risperidone for 
autism, the authors report that, in total, 27\% of 95 parents retrospectively stated that treatment arm assignment had been based on the individual needs of the child. Of the 88 parents of children who had not previously received care from the investigators, this therapeutic misconception was $28 \%$. Unexpectedly, the rate of misconception for seven parents of children who had been previously treated by the investigators was only $14 \%$, half the rate of misconception of among families new to the researchers. These findings may be of limited significance given the small sample size of the previously treated group.

Ward describes a high rate of therapeutic misconception among 27 parents of babies offered various greaterthan-minimal-risk studies at three neonatal intensive care units on the Mid-Atlantic coast and offers insight into how and why parents harbor therapeutic misconception. ${ }^{27}$ Using qualitative descriptive analysis, the article states that parents perceived the hospital setting and the research protocols as "chaos," which led them to feel vulnerable. Nevertheless, parents were able to cope with that chaos and vulnerability by finding a way to take "control" of their difficult situations. One way parents gained control of the situation was by reconceptualizing the proposed studies as interventions they could choose to provide the best possible treatment for their babies.

The mother of a prematurely born child in this study compared forgoing clinical research to parental negligence: "If your child started choking, would you just sit there and not do anything or would you try to help your child?" Another mother said that she "felt empower(ed) that we were doing something good for our child." Thus, research provided a way for some parents to actively influence their children's health outcomes. ${ }^{27}$

Unfortunately, this reconceptualization led the parents to underestimate the implication of randomization (ie, that the experimental treatment was not known to be better than the control). Parents had created the therapeutic misconception as a means of coping with the illness of their babies and the chaos they perceived in the hospital setting. The authors speculate that in minimal-risk trials or nontherapeutic trials on healthy children, parents may have very different emotions.

\section{State of shock and emotional implications of giving permission}

Ward's paper, "Chaos, vulnerability, and control: parental beliefs about neonatal clinical trials," explores the guilt, fear, and motivations of parents of babies who had been enrolled in neonatal clinical trials. ${ }^{27}$ Many of the parents acknowledged feigning understanding of the protocol when they signed the permission document because they were struggling to quickly learn about their child's diagnosis, proposed research, and the neonatal intensive care setting. Kodish et al's ${ }^{28} 1998$ pilot report on permission and assent in leukemia clinical trials suggests that parental stress is the primary obstacle to understanding information about clinical trials. Both subjects and investigators described the state of shock that hinders parents from making thoroughly informed decisions.

In a survey of 109 parents who had participated in permission-assent conferences for 25 pediatric clinical trials across Europe, $30 \%$ stated that they were somewhat or very nervous at the time of signing the permission document; $18 \%$ were unable to concentrate when they signed the permission document. ${ }^{29}$ Seventy percent of respondents in the same survey felt that they understood the project protocols to which they gave permission. In an editorial on the difficulties of informing parents and children about research, Dawson et $\mathrm{a}^{30}$ described emotional state after a difficult childbirth as impedimentary to good understanding, and Kupst et $\mathrm{al}^{31}$ (2003) found that $70 \%$ of parents of 17 newly diagnosed child cancer patients reported that a state of shock inhibited their understanding of presented research. Of the same group, parents of only five of the 13 children who had begun randomized treatment protocols correctly identified that the treatments were chosen at random. According to these authors, the state of shock inhibits parents' understanding of randomization.

Some ethicists have suggested that parents should not be expected to deal with the decision to participate in research when they are struggling to comprehend a difficult diagnosis. An anonymous author in Lancet cites almost universal poor understanding of neonatal clinical trials because of a state of emotional shock, suggesting that informed permission for neonates is a sham. "[I]n the absence of evidence from controlled trials, clinical decisions are often made randomly in a haphazard way. "Why, then," the author asks, "are we so concerned [as to require permission] when the randomness is formalized in a trial?" 32 "Your baby is in a trial" concludes that the physician is ultimately responsible for proper research conduct, and asking parents to give permission for a protocol that they do not understand serves only to increase their anxiety.

Harth and Thong describe other aspects of clinical trials that parents have trouble understanding. ${ }^{33}$ For instance, $88 \%$ of 64 parents interviewed after their children had completed a randomized, double-blind, placebo-controlled 
trial of ketotifen for asthma did not realize that the drug trial tests for safety as well as efficacy. Additionally, $81 \%$ of these parents did not understand that permission documents are intended to protect patients' and parents' rights; $40 \%$ of these parents assumed that permission forms are to protect doctors from litigation. Incidentally, "Your baby is in a trial" calls this assumption correct and suggests that permission documents are a means by which doctors and institutional review boards shirk responsibility for ensuring safety in their clinical trials.

In an essay about the difficulties of obtaining valid parental permission for pediatric clinical trials, Mason pointed out that when investigators explain that they do not know the best treatment for a child (as is the case in a randomized clinical trial), parents may lose confidence in the medical team, further exacerbating their stress. ${ }^{34}$ Mason also argued that ethics committees or institutional review boards assure that research projects are safe, with or without parental permission, and describes the permission process in neonatal trials as an "elaborate ritual." Mason did not discuss ways that oversight committees promote safety in clinical trials (eg, by assessing risk level and reviewing consent and permission documents and making suggestions for their improvement). Other authors have joined "Your baby is in a trial" in calling for reducing the regulatory requirements for parental permission. Dawson et al warned that regulatory difficulty for pediatric investigators impedes the recruitment of children into clinical trials, which is against the best interests of children in general, and in a survey of 107 neonatologists, Mason et al reported that nearly half of the respondents felt that the requirement to obtain informed permission prevents useful neonatal research. ${ }^{30,35}$

On the other hand, even though obtaining meaningful permission from distressed parents is difficult, it is not impossible. Respect for autonomy as described by Beauchamp and Childress requires that investigators make a good-faith effort to obtain consent from their adult subjects or permission from parents and assent from pediatric subjects. ${ }^{36}$ Frustration with the permission process for pediatric research should inspire efforts to make clinical research easier for parents and children to understand. Furthermore, parents often describe the opportunity to decide about research participation, even under emotional stress, as an essential fulfillment of their social and legal responsibility to act as protectors of their children and to make choices in their best interest. ${ }^{27}$ Shilling and Young's narrative review of the literature on why parents chose to enroll their children in clinical research described the emotional journey of parents considering trial participation for their children. ${ }^{37}$ They suggested that investigators can improve parental satisfaction with the medical experience by validating parental choices about research participation whether parents decline research or opt for enrollment.

Tight time constraints do not reduce the meaningfulness of parental decision making. Snowdon et al interviewed 62 parents after they had permitted or refused one of four antenatal or neonatal clinical trials at one of eight hospitals in Great Britain. ${ }^{38}$ They qualitatively report that when decisions about research needed to be made quickly in a maternity or neonatal intensive care ward, most of the parents reported feeling rushed but did not find the rush problematic, despite investigator concerns that they would.

In a retrospective survey of 29 parents who had been offered clinical trials for their neonates, Burgess et al reported that $38 \%$ noted that the permission process added "stress to an already stressful situation." 39 Researchers explained that institutional review boards and principal investigators take special care to try to ensure the safety of human subjects and then asked parents if they felt researchers should save them the stress of having to decide about trial participation. Despite knowing this, 93\% of parents were opposed or strongly opposed to moving the responsibility to decide from the parents to the doctors. In a randomized trial of pulmonary function testing on sick newborns, 83 of 99 parents said they would have been unhappy to have foregone the permission process. ${ }^{40}$ Similarly, only $15 \%$ of 64 parents of children in research interviewed by Harth et al reported that permission was unnecessary "because they would follow the doctor's advice anyways." ${ }^{33}$ As previously noted, most parents gain a sense of ownership and control through their participation in the permission-assent process; they want to be included in the decision.

\section{Child-subject assent, understanding, and adolescent attitudes}

Evidence exists that pediatric subjects also harbor a therapeutic misconception. Koelch et al described therapeutic misconception among children ages 7 to 19 who were enrolled in randomized, placebo-controlled trials of atomoxetine or methylphenidate for attention deficit/hyperactivity disorder. ${ }^{41}$ They also noted that randomization and placebo uses were poorly understood. According to the authors, 13 of the 19 children in this study and one-third of their parents had 
a therapeutic misconception about the purpose of the trials. Furthermore, legal minors thought the purpose of the placebo was to see if the subjects really needed treatment.

Almost two decades earlier, Abramovitch et al described children's difficulty understanding the investigative nature of research. ${ }^{42}$ They studied 190 children age 5 to 12 years who assented to one of four nontherapeutic psychology studies. According to the authors, $10 \%$ of the 5 - to 6 -yearolds, $27.5 \%$ of the 7 - to 8 -year-olds, and $61.4 \%$ of the 9 - to 11 -year-olds were able to correctly identify the goals of the study. The report did not specify the details of the "incorrect" responses about the goals of research, so the presence of a therapeutic misconception, while implied, was not explicitly described.

Ondrusek et al were especially interested in the age at which children become able to understand research and give meaningful assent. ${ }^{43}$ They interviewed 18 children aged 5 to 18 years who had participated in a nontherapeutic nutritional study that included two blood draws and sitting still while breathing into a plastic hood for an extended period of time. Age influenced understanding of both the goals of the study and of the risks and benefits to the subjects. None of children less than 9 years old could describe the goals of the study, and all but one of the older children were able to correctly describe that researchers were trying to learn about caloric needs so that they could help sick children in the future.

Similarly, all of the children 9 years or older correctly identified the benefit to the subject as "none" or "learning about the amount of calories my body needs to function," and were more likely to identify the needle-stick as a possible harm of the study. Children less than 9 years old, though, said they did not know what the benefits would be, or they said they would "have fun" from being in the study.

A correlation of understanding with age was described also by Abramovitch et al. Sixty-two percent of 5- to 6-yearolds were able to describe the basic procedures of the study, while $100 \%$ of 9 - to 11 -year-olds were able to do the same. For children aged 7 to 8 years, an intermediate proportion, $87.5 \%$, were able to describe basic study procedures. ${ }^{42}$

Tait et al's report on understanding of nontherapeutic research associated with elective procedures indicated that children less than 11 years old have a "limited understanding" ( $\mathrm{n}=102) .{ }^{16}$ In that study, $7.7 \%$ of 7 - to 10 -year-olds had complete understanding of study protocols, compared to $40 \%$ of 11 - to 14 -year-olds and $63.6 \%$ of 15 - to 18 -yearolds. The group of children younger than 11 years old had similarly low proportions of complete understanding of the benefits to self and to others and the alternatives to study participation.

Younger child-subjects do not seem to appreciate the right to withdraw from studies without negative consequences. When Abramovitch et al asked subjects "What happens if you want to stop being in the study?" $38 \%$ of 5 - to 6 -year-olds, $45 \%$ of 7 - to 8 -year-olds, and $79.5 \%$ of 9 - to 11 -year-olds correctly stated that they could simply leave or ask to stop. ${ }^{42}$ Slightly less reassuring numbers were reported by Ondrusek et al, who state that $25 \%$ of 5- to 9 -year-olds and $78 \%$ of 10 - to 18 -year-olds had a good understanding of the right to withdraw. ${ }^{43}$ Ondrusek et al also describe child-subjects' belief that investigators and parents would be upset if the subjects decided to withdraw from the study. This belief was held by most children, regardless of age. Even children who recalled being told that they could withdraw at will indicated that withdrawing might upset parents or researchers. This feeling may correlate with the societal role of children, who typically are expected to comply with the suggestions of authority figures and parents. Children often have limited authority over their daily schedules and activities, especially with regard to health care, so it is not surprising that they would perceive parental or investigator pressure to participate in the study.

Child-subjects also had difficulty understanding the concept of confidentiality. A subset of 58 of Abramovitch et al's subjects was questioned specifically about the confidentiality of subject responses to investigators' questions. Forty-one percent of 8 - to 9 -year-olds and $50 \%$ of 10 - to 11 -year-olds thought that the experimenter would divulge the children's answers to parents who asked for them, even though the children were told that only the experimenter would know the children's answers. ${ }^{42}$

For adolescents, the capacity to understand is generally assumed to approach that of adults, so much of the literature on adolescent assent focuses on judgment and values rather than understanding. Scherer and Reppuci described parental influence over 40 healthy adolescents' attitudes about assenting to treatment for various hypothetical maladies. ${ }^{44}$ Each vignette was presented in three stages. In the first stage, two possible interventions were presented, and the adolescents were asked to pick the one they preferred. Stage two described the same diagnosis, but a parent expressed preference for the option the subject had not chosen, and the adolescent was asked if in the new scenario they would have chosen the other option. In stage three a stronger parental 
preference was described, and this time the parent tried to coerce the subject into choosing the unselected treatment option by asserting parental power. The adolescents reported that they would usually defer to parents' preference; however, they were less likely to defer if the proposed intervention was serious, (eg, renal transplant versus wart removal). Even though this study was about medical treatments and not research, the elements required for consent or assent to the intervention may be similar.

In a more recent but related study, Brody et al presented a hypothetical asthma research study to 37 asthmatic adolescents and their parents. ${ }^{45}$ Because Brody et al only used one hypothetical study, the effect of variable parental preference or intervention severity on adolescent attitudes could not be determined. Ironically, both adolescents and their parents claimed the ultimate responsibility for the decision to join a clinical trial. Initially, adolescents and their parents agreed about whether to participate in a pediatric clinical trial $74 \%$ of the time. Upon questioning of the decision-discordant parent-child pairs, most parents expected that their child would acquiesce to parental wishes; however, adolescents stated that their parents should respect their decisions about their own health care. Brody et al called for further research to characterize how these disagreements are resolved in nonhypothetical cases. Not surprisingly, adolescents who are beginning to assume the responsibility of an independent adult express a strong desire to be part of the decision to participate in research.

Physician-investigators should account for child and physician attitudes and understanding of clinical research and the nature of assent. Ungar et al hold that adolescents should cosign the permission form with their parents as a sign of the importance of the adolescent assent. ${ }^{46}$ For younger children who do not understand the symbolic nature of the signature, they suggest physicians document assent or dissent in the medical record with a brief note.

\section{Interventions to improve understanding} Cultural attitudes, learning style, and intelligence profile may also impact a subject's understanding. These influences are numerous, complex, and intangible. Some researchers have considered interventions to increase subject understanding.

Most importantly, parent-surrogates and child-subjects cannot be expected to understand information that they are not given. Several studies have evaluated exactly what information is conveyed at permission-assent conferences and how it is presented. Kodish et al audiotaped 140 permission-assent conferences for randomized leukemia treatment trials. ${ }^{19}$ Parents were then interviewed shortly after the conference. In the postconference interviews, only 50\% of parents understood that treatment assignment in the study would be random. Indeed, randomization was not mentioned in $17 \%$ of the permission-assent conferences. Kodish et al also reported that treatment options outside a trial were discussed in $89 \%$ of these conferences. Investigators offered to answer questions about the proposed clinical trial in only $59 \%$ of permission-assent conferences. Ironically, the $83 \%$ of parents who were explicitly told about randomization at the permission-assent conference did not seem to understand the concept of randomization any better than the $17 \%$ of parents who were not told about randomization, suggesting that other factors, such as a therapeutic misconception or emotional stress, might have been more important than disclosure.

Simon et al reported additional data on the same subjects. ${ }^{19,47}$ Specifically, only $37 \%$ of parents were told why their child qualified for the particular clinical trial. Parents were told that participation was voluntary and that they could withdraw from the study at any time in $96 \%$ and $71 \%$ of permission-assent conferences, respectively. By contrast, at the postconference interview $18 \%$ of parents thought that their child had to enroll in the study to receive treatment, and $20 \%$ reported that they would not be able to withdraw at will. The possible complications of study participation were compared to the complications associated with the standard treatment in only $3 \%$ of the permission-assent conferences, but in none of the conferences was any numerical estimation of the risk of complications provided. Even more surprising was that in none of the permission-assent conferences were the inconveniences of study participation compared to the inconveniences of the standard treatment. It is possible that some of this information was not disclosed at the permission-assent conference because it was not known; still, it seems the information would be both relevant and desirable to parents.

In an exposé on permission and assent in anesthesiology research, Erb et al suggested that investigators explicitly tell parents and children that clinical trials are not primarily devoted to treatment, but rather to improving the scientific community's knowledge. ${ }^{48}$ They asserted that such frankness is necessary to reduce therapeutic misconception rates. Understanding of the goals of research is essential for valid permission; however, such a direct statement is only appropriate if it is true. In fact, many clinical trials 
have the dual purpose of treating patients and improving medical knowledge (ie, both goals are equally important). For many parents struggling to deal with the diagnosis of a serious childhood illness, the goal of treating the child will seem the most relevant to parents and should not be deemphasized.

\section{Shared and staged consent}

The manner in which information is presented is as important as the information itself. Based on questionnaire responses of 89 clinicians who regularly enroll subjects in Children's Oncology Group trials, Simon et al advised giving less information at one time to parents and children so as not to overwhelm them; however, this recommendation should be approached cautiously. ${ }^{49}$ Most clinicians underestimate how much information parents want to receive and most parents report preferring more information than they received from the clinician. ${ }^{28,50}$ Similarly, Kupst et al report that $50 \%$ to $66 \%$ of 20 parents offered clinical trials for their children after they were diagnosed with cancer found the discussion of alternatives to research insufficient. ${ }^{31}$

If, as Simon et al suggests, the information presented at one time is to be limited, multiple episodes of information delivery could provide the amount of information that parents want and need in order to be adequately informed. Indeed, several authors recommend the use of a developing continuum of information delivery, or "staged consent" to deliver more information than could be given at one permission-assent conference. ${ }^{18,19,25,28,49-52}$ In "staged consent" physician-investigators base the timing, scope, and character of verbally presented information on the child's and parent's age, current understanding, and ability to process the information. The need for more information is constantly reassessed. Short quizzes for subjects may help researchers know what information needs to be reinforced at the next permission-assent conference.

Massimo et al emphasized the needs and knowledge base of the child in the permission-assent process by calling the whole process "shared consent," implying that the child is an important stakeholder in the process. ${ }^{53}$ Simon found the goals of permission and assent sometimes mutually impeding. ${ }^{47}$ In these cases, he suggested investigators consider separate meetings for child-tailored and parent-tailored information delivery. Dawson et al state much informational material is inappropriate for children. ${ }^{30}$ They therefore suggested that children should be involved in the production of informational forms and booklets for other children. They also noted that pamphlets and internet resources for children could be modeled after successful websites and booklets developed to help children understand chronic diseases such as asthma and cancer.

Postlethwaite et al describe a well-devised stagedconsent procedure with parental knowledge assessments and at least two information-delivery conferences. ${ }^{54}$ More meetings were held if the parents did not have adequate understanding after just two meetings. Eighty percent of the parents in 14 families were found to have "good" or "very good" understanding of the study details after the modified permission-assent process; however, understanding in these families was not directly compared to understanding in families that had more traditional permission-assent processes.

Multiple sessions of information delivery do not necessarily require that the investigator be present at each one. Yamokoski et al report that $77 \%$ of 35 parents of children recently diagnosed with leukemia found an anticipatory meeting with a nurse-educator before the permission-assent conference to help with understanding. ${ }^{55}$ Unfortunately, the study did not actually evaluate parental understanding and compare it to the understanding of parents who did not participate in an anticipatory guidance session.

Some authors have expressed dissatisfaction with certain aspects of staged and shared consent processes, which will likely continue to evolve as researchers find more ways to improve parent-surrogate and child-subject understanding and satisfaction. Massimo et al found that the requirement of a second permission document at the time of randomization, as is often required by research oversight committees, impedes mutual understanding and increases parental anxiety about a protocol they had already permitted. ${ }^{23,53}$ André responded to the idea of shared consent with concern that its practical application could delay needed treatments for children. He concludes

$$
\begin{aligned}
& {[\ldots] \text { medical students and doctors should also receive }} \\
& \text { information on the ethical and pragmatic risks, as well as } \\
& \text { the limits associated with shared consent, to promote and } \\
& \text { preserve the child's best interests. }{ }^{56}
\end{aligned}
$$

\section{Alternative methods of communication}

Some researchers have systematically tested various ways of giving information to determine if they could improve understanding. For example, knowing that investigators describe randomization many different ways, Kodish et al also studied whether the way researchers presented 
randomization (eg, coin flip, computer-generated numbers, center office, rolling the dice) influenced understanding. ${ }^{19,57}$ No one description was better understood by parents or children.

Tait et al, recognizing that many consent forms are difficult to read, tried a modified consent form with 1) simpler language, 2) cartoon drawings, and 3) bullet points (as opposed to paragraphs). ${ }^{58,59}$ The modified form improved the mean overall parental understanding score of a hypothetical pediatric study from $6.6 \pm 1.6$ of a possible 10 points to $7.6 \pm 1.3$ $(P<0.001)$, especially for parents with reading abilities at less than the 9th-grade level ( $4.3 \pm 1.00$ versus $26.7 \pm 1.9, P=0.003)$. When shown both the standard and modified forms, parents preferred the modified form.

Barfield and Church are currently assessing the use of "interactive computer technologies" as another tool for improving the assent-permission process in pediatric research. ${ }^{24}$ Further research on mediums of information presentation seems necessary given the equivocal results presented above. Indeed, a Cochrane review which considered some of the above articles did not find audiovisual presentations to consistently increase subject understanding.

Further research in the form of randomized controlled trials was recommended. Such research should include consideration of varying levels of understanding and education, different ages, and diverse cultural groups. ${ }^{60}$

Better data would certainly inform better ethical guidelines.

\section{Educating the public and practitioners}

Sherman et al used a wait-list-control group to compare 27 pediatric interns in a randomized, crossover trial of a 1-hour class on pediatric informed consent. ${ }^{61}$ The class consisted of a lecture, video presentation, and discussion. Completers scored better than noncompleters on a validated instrument measuring "patient autonomy and empowerment" (17.7 \pm 1.5 versus $16.4 \pm 2.3, P<0.01)$, "physician obligations" (21.3 \pm 2.9 versus $19.9 \pm 3.6, P<0.05)$, and "collaboration/sharing in medical decision making" ( $8.4 \pm 1.0$ versus $7.3 \pm 1.7, P<0.01)$. This intervention approaches the formal training in facilitation of permission-assent conferences that Kodish et $\mathrm{al}^{28}$ and Mason and Allmark ${ }^{35}$ call for; however, the intervention dealt with general informed permission and assent and was not specific to research. One limitation was that there was no long-term follow-up to see how the residents retained presented information.

Erb et al reviewed permission and assent for pediatric anesthesia research. The authors stated that parents and potential subjects, as members of a well-educated public, should learn about the basic nature of scientific research "well in advance of admission to the hospital." 48 Burgess et al surveyed 29 parents of babies in various neonatal clinical trials. ${ }^{39}$ The primary response of many parents to the openended questions about how the permission process could be improved was that information about research should be made available before parents present to the hospital for delivery.

Spencer et al developed a four-page informational pamphlet about general clinical research and piloted it on 37 inpatients and outpatients who were not being recruited into research projects. ${ }^{62}$ The booklet described ethics committees, goals of clinical research, clinical equipoise, informed consent, and parental permission and child assent for pediatric research. Recipients were contacted by telephone to assess their understanding of the document and generate suggestions for its improvement. Despite not being invested in the prospect of becoming clinical research subjects, recipients showed good understanding of the material presented in the pamphlet (eg, 97\% of recipients correctly stated that parents give permission for their children to participate in research and $73 \%$ correctly stated that they would not know if a novel treatment being studied would be better than the standard of care or not). At the suggestion of some respondents, Spencer et al incorporated examples of research scenarios in the pamphlet, and it was approved for wide distribution at their institution's outpatient clinics in hopes of improving the public's knowledge about clinical research.

\section{Conclusion}

Most authors advocate continuous delivery of clinical-trials information throughout the trial process. This is often called "staged consent" because multiple permission and assent documents may be used at multiple conferences. Investigators should continually reassess understanding and completely present relevant facts to child-subjects and parent-surrogates as they are ready to understand them. Families should not be expected to understand important concepts if they are not presented.

Scientifically rigorous research is needed into the use of alternative mediums of information delivery. Thus far, some researchers have found them useful, but others have failed to demonstrate that they improve understanding. Children should be involved in the development of informationdelivery instruments to ensure that such tools are, in fact, child-appropriate. 
Education of medical trainees may help to improve their ability to facilitate permission-assent conferences, and education of the public about the differences between clinical research and noninvestigative interventions may help to alleviate misunderstanding when specific trials are presented.

Finally, since the scientific revolution, systematic research on child-subjects has dramatically evolved, not only with regard to our scientific methodology but also with regard to our appreciation for the vulnerability and preciousness of pediatric subjects. Permission and assent policies may seem overbearing in their regulatory nature and incapable of achieving their stated goal, which is to ensure valid, informed permission and assent where appropriate for each pediatric child-subject. Still, these policies represent our efforts and remind us of our duty - to protect pediatric subjects. Physician-investigators should continue to reassess both the information needs of their subjects and the ethical requirements of the permission-assent process.

\section{Acknowledgments}

The authors gratefully acknowledge Feng-Chang Lin, MS, and Michael Evans for their help with the statistical analysis of the results.

\section{Disclosure}

The authors report no conflicts of interest in this work.

\section{References}

1. National Research Council. The Ethical Conduct of Clinical Research Involving Children. Washington, DC: The National Academies Press, 2004.

2. Diekema DS. Conducting ethical research in pediatrics: a brief historical overview and review of pediatric regulations. $J$ Pediatr. 2006; 149(Suppl 1):S3-S11.

3. Anonymous. Ruthless practices in Vienna Hospitals. The New York Times 1899:2.

4. Preussisches Ministerium fuer Religion, Unterricht und Medizin: Instruktionen fuer die Direktoren der Krankenanstalten und andere medizinische Einrichtungen [Prussian ministry of religion, lessons and medicine: Instructions for the managers of the sick person's institutions and other medical equipment]. 1900:188-189. German.

5. Beecher HK. Ethics and clinical research. N Engl J Med 1966; 274(24):1354-1360.

6. Krugman S. The Willowbrook hepatitis studies revisited: ethical aspects. Rev Infect Dis. 1986;8(1):157-162.

7. Unguru Y, Coppes MJ, Kamani N. Rethinking pediatric assent: from requirement to ideal. Pediatr Clin North Am. 2008;55(1):211-222, xii.

8. Chesney RW, Christensen ML. Changing requirements for evaluation of pharmacologic agents. Pediatrics. 2004;113(Suppl 4):1128-1132.

9. Bartholome WG. A new understanding of consent in pediatric practice: consent, parental permission, and child assent. Pediatr Ann. 1989;18(4):262-265.

10. Committee on Bioethics, American Academy of Pediatrics. Informed consent, parental permission, and assent in pediatric practice. Pediatrics 1995;95(2):314-317.
11. United States National Institutes of Health. NIH Policy and Guidelines on the Inclusion of Children as Participants in Research Involving Human Subjects. 1998.

12. Ross JA, Olshan AF. Pediatric cancer in the United States: the Children's Oncology Group Epidemiology Research Program. Cancer Epidemiol Biomarkers Prev. 2004;13(10):1552-1554.

13. The Code of Federal Regulations. Title 45: Public Welfare, Part 46: Protection of Human Subjects. 2009.

14. Chesney RW. Children as clinical research subjects. J Pediatr. 2005; 146(5):579-580.

15. The University of Wisconsin-Madison Graduate School, Human Research Protection Program. Guidance: Informed Consent. 2007.

16. Tait AR, Voepel-Lewis T, Malviya S. Do they understand? (part II): assent of children participating in clinical anesthesia and surgery research. Anesthesiology. 2003;98(3):609-614.

17. Tait AR, Voepel-Lewis T, Malviya S. Do they understand? (part I): parental consent for children participating in clinical anesthesia and surgery research. Anesthesiology. 2003;98(3):603-608.

18. Simon CM, Siminoff LA, Kodish ED, Burant C. Comparison of the informed consent process for randomized clinical trials in pediatric and adult oncology. J Clin Oncol. 2004;22(13):2708-2717.

19. Kodish E, Eder M, Noll RB, et al. Communication of randomization in childhood leukemia trials. JAMA. 2004;291(4):470-475.

20. Hazen RA, Drotar D, Kodish E. The role of the consent document in informed consent for pediatric leukemia trials. Contemp Clin Trials. 2007;28(4):401-408.

21. Appelbaum P, Roth L, Lidz C. The Therapeutic Misconception: Informed Consent in Psychiatric Research. Intl J Law Psychiatry. 1982;5:319-329.

22. Edwards SJ, Lilford RJ, Braunholtz DA, Jackson JC, Hewison J, Thornton J. Ethical issues in the design and conduct of randomised controlled trials. Health Technol Assess. 1998;2(15):i-vi, 1.

23. Massimo LM, Wiley TJ. Randomization, informed consent and physicians' communication skills in pediatric oncology: a delicate balance. Bull Cancer. 2005;92(12):E67-E69.

24. Snowdon C, Garcia J, Elbourne D. Making sense of randomization; responses of parents of critically ill babies to random allocation of treatment in a clinical trial. Soc Sci Med. 1997; 45(9):1337-1355.

25. Barfield RC, Church C. Informed consent in pediatric clinical trials. Curr Opin Pediatr. 2005;17(1):20-24.

26. Vitiello B, Aman MG, Scahill L, et al. Research knowledge among parents of children participating in a randomized clinical trial. $J \mathrm{Am}$ Acad Child Adolesc Psychiatry. 2005;44(2):145-149.

27. Ward FR. Chaos, vulnerability and control: parental beliefs about neonatal clinical trials. J Perinatol. 2009;29(2):156-162.

28. Kodish ED, Pentz RD, Noll RB, Ruccione K, Buckley J, Lange BJ. Informed consent in the Childrens Cancer Group: results of preliminary research. Cancer. 1998;82(12):2467-2481.

29. Franck LS, Winter I, Oulton K. The quality of parental consent for research with children: a prospective repeated measure self-report survey. Int J Nurs Stud. 2007;44(4):525-533.

30. Dawson A, Spencer SA. Informing children and parents about research. Arch Dis Child. 2005;90(3):233-235.

31. Kupst MJ, Patenaude AF, Walco GA, Sterling C. Clinical trials in pediatric cancer: parental perspectives on informed consent. J Pediatr Hematol Oncol. 2003;25(10):787-790.

32. Anonymous. Your baby is in a trial. Lancet. 1995; 345(8953):805-806.

33. Harth SC, Thong YH. Parental perceptions and attitudes about informed consent in clinical research involving children. Soc Sci Med. 1995;41(12):1647-1651.

34. Mason S. Obtaining informed consent for neonatal randomised controlled trials - an "elaborate ritual"? Arch Dis Child Fetal Neonatal Ed. 1997;76(3):F143-F145.

35. Mason SA, Allmark PJ. Obtaining informed consent to neonatal randomised controlled trials: interviews with parents and clinicians in the Euricon study. Lancet. 2000;356(9247):2045-2051. 
36. Beauchamp T, Childress J. Principles of Biomedical Ethics, Oxford: Oxford University Press; 1994.

37. Shilling V, Young B. How do parents experience being asked to enter a child in a randomised controlled trial? BMC Med Ethics. 2009;10:1.

38. Snowdon C, Elbourne D, Garcia J. "It was a snap decision": parental and professional perspectives on the speed of decisions about participation in perinatal randomised controlled trials. Soc Sci Med. 2006;62(9): 2279-2290.

39. Burgess E, Singhal N, Amin H, McMillan DD, Devrome H. Consent for clinical research in the neonatal intensive care unit: a retrospective survey and a prospective study. Arch Dis Child Fetal Neonatal Ed. 2003;88(4):F280-F285; discussion F285-F286.

40. Stenson BJ, Becher JC, McIntosh N. Neonatal research: the parental perspective. Arch Dis Child Fetal Neonatal Ed. 2004;89(4): F321-F323.

41. Koelch M, Singer H, Prestel A, Burkert J, Schulze U, Fegert JM. “... because I am something special" or "I think I will be something like a guinea pig": information and assent of legal minors in clinical trials - assessment of understanding, appreciation and reasoning. Child Adolesc Psychiatry Ment Health. 2009;3(1):2.

42. Abramovitch R, Freedman JL, Thoden K, Nikolich C. Children's capacity to consent to participation in psychological research: empirical findings. Child Development 1991;62(5):1100-1109.

43. Ondrusek N, Abramovitch R, Pencharz P, Koren G. Empirical examination of the ability of children to consent to clinical research. $J$ Med Ethics. 1998;24(3):158-165.

44. Scherer DG, Reppuci ND. Adolescents' capacities to provide voluntary informed consent: the effects of parental influence and medical dilemmas. Law Hum Behav. 1988;12(2):123-141.

45. Brody JL, Scherer DG, Annett RD, Pearson-Bish M. Voluntary assent in biomedical research with adolescents: a comparison of parent and adolescent views. Ethics Behav. 2003;13(1):79-95.

46. Ungar D, Joffe S, Kodish E. Children are not small adults: documentation of assent for research involving children. J Pediatr. 2006;149(Suppl 1): S31-S33.

47. Simon C. Child assent and parental permission for clinical research some considerations. Bioethics Forum. 2002;18(3-4):36-42.

48. Erb TO, Schulman SR, Sugarman J. Permission and assent for clinical research in pediatric anesthesia. Anesth Analg. 2002;94(5):1155-1160.

49. Simon C, Eder M, Raiz P, Zyzanski S, Pentz R, Kodish ED. Informed consent for pediatric leukemia research: clinician perspectives. Cancer. 2001;92(3):691-700.
50. Eder ML, Yamokoski AD, Wittmann PW, Kodish ED. Improving informed consent: suggestions from parents of children with leukemia. Pediatrics. 2007;119(4):e849-e859.

51. Rossi WC, Reynolds W, Nelson RM. Child assent and parental permission in pediatric research. Theor Med Bioeth. 2003;24(2):131-148.

52. Kuther TL, Posada M. Children and adolescents' capacity to provide informed consent for participation in research. Adv Psychol Res. 2004:32:163-173.

53. Massimo LM, Wiley TJ, Casari EF. From informed consent to shared consent: a developing process in paediatric oncology. Lancet Oncol. 2004;5(6):384-387.

54. Postlethwaite RJ, Reynolds JM, Wood AJ, Eminson DM. Recruiting patients to clinical trials: lessons from studies of growth hormone treatment in renal failure. Arch Dis Child. 1996;74(1):91.

55. Yamokoski AD, Hazen RA, Kodish ED. Anticipatory guidance to improve informed consent: a new application of the concept. J Pediatr Oncol Nurs. 2008;25(1):34-43.

56. Andre N. Involving children in paediatric oncology decision-making. Lancet Oncol. 2004;5(8):467.

57. Sabik L, Pace CA, Forster-Gertner HP, et al. Informed consent: practices and views of investigators in a multinational clinical trial. IRB. 2005;27(5):13-18.

58. Tait AR, Voepel-Lewis T, Malviya S, Philipson SJ. Improving the readability and processability of a pediatric informed consent document: effects on parents' understanding. Arch Pediatr Adolesc Med. 2005;159(4):347-352

59. Grossman SA, Piantadosi S, Covahey C. Are informed consent forms that describe clinical oncology research protocols readable by most patients and their families? J Clin Oncol. 1994;12(10):2211-2215.

60. Ryan RE, Prictor MJ, McLaughlin KJ, Hill SJ. Audio-visual presentation of information for informed consent for participation in clinical trials. Cochrane Database Syst Rev. 2008:CD003717.

61. Sherman HB, McGaghie WC, Unti SM, Thomas JX. Teaching pediatrics residents how to obtain informed consent. Acad Med. 2005; 80(Suppl 10):S10-S13.

62. Spencer SA, Dawson A, Rigby C, Leighton N, Wakefield J. Informing parents about research: evaluation of an information leaflet. Qual Prim Care. 2004;12:37-46.
Open Access Journal of Clinical Trials

\section{Publish your work in this journal}

The Open Access Journal of Clinical Trials is an international, peerreviewed, open access journal publishing original research, reports, editorials, reviews and commentaries on all aspects of clinical trial design, management, legal, ethical and regulatory issues, case record form design, data collection, quality assurance and data auditing

\section{Dovepress}

methodologies. The manuscript management system is completely online and includes a very quick and fair peer-review system, which is all easy to use. Visit http://www.dovepress.com/testimonials.php to read real quotes from published authors. 